\title{
Direct Common Carotid Artery Puncture: Rescue Mechanical Thrombectomy Strategy in Acute Ischemic Stroke
}

\author{
Cetin Kursad Akpinar, $\mathrm{MD}^{1}$, Erdem Gurkas, $\mathrm{MD}^{2}$, Ozlem Aykac, $\mathrm{MD}^{3}$, Yusuf Inanc, $\mathrm{MD}^{4}$, \\ Semih Giray, $\mathrm{MD}^{4}$, Atilla Ozcan Ozdemir, $\mathrm{MD}^{3}$ \\ ${ }^{1}$ Deparment of Neurology, Samsun Training and Research Hospital, Samsun, Turkey \\ ${ }^{2}$ Department of neurology, Lütfi Kırdar Training and Research Hospital, İstanbul, Turkey \\ ${ }^{3}$ Department of Neurology, Medical Faculty, Eskisehir Osmangazi University, Eskisehir, Turkey \\ ${ }^{4}$ Department of Neurology, Medical Faculty, Gaziantep University, Gaziantep, Turkey
}

Purpose: In a minority of cases, the transfemoral approach cannot be performed due to unfavorable anatomical barriers. In such cases, direct common carotid artery puncture (DCCAP) is an important alternative for rescue mechanical thrombectomy. The purpose of this study was to evaluate the efficacy and safety of DCCAP in patients with an unaccessible femoral route for mechanical thrombectomy.

Materials and Methods: This is a retrospective study using data in the Turkish Interventional Neurology Database recorded between January 2015 and April 2019. Twenty-five acute stroke patients treated with DCCAP were analyzed in this study. Among 25 cases with carotid puncture, 4 cases were excluded due to an aborted thrombectomy attempt resulting from unsuccessful sheath placement.

Results: Patients had a mean age of $69 \pm 12$ years. The average National Institutes of Health Stroke Scale score was $16 \pm 4$. Successful revascularization (modified Thrombolysis In Cerebral Infarction 2b-3) rate was 86\% (18/21), and 90-day good functional outcome rate (modified Rankin Scale 0-2) was 38\% (8/21).

Conclusion: DCCAP is a rescue alternative for patients with unfavorable access via the transfemoral route. Timely switching to DCCAP is crucial in these cases.

Key Words: Stroke; Thrombectomy; Treatment outcome; Common carotid artery

\section{INTRODUCTION}

Mechanical thrombectomy is the most effective treatment method that should be applied within the shortest time to suitable patients in acute stroke resulting from large vessel occlusion. ${ }^{1-3}$ Fast and complete recanalization of the occluded vessel is very important for achieving a good clinical outcome. ${ }^{1,2}$ Factors such as advanced age, femoral and/or iliac artery tortuosity or occlusion, unfavorable aortic arch (Type 2-3, bovine arch), supra-aortic vessel tortuosity, and supra-aortic vessel osteal lesions delay access to the occluded vessel and prolong procedure time, and thus recanalization time, resulting in poor functional outcome. ${ }^{3}$ In cases where the transfemoral approach is not possible, radial, brachial, and common carotid arteries (CCA) are alternative pathways that can be used. ${ }^{4,5}$

\section{Correspondence to:}

Cetin Kursad Akpinar, MD

Stroke Center, Department of Neurology, Samsun Training and Research Hospital, Kışla, Barış Blv. No: 199, 55090 Ilkadim, Samsun, Turkey

Tel: +90-5422262605

Fax: +90-3623121919

E-mail:dr_ckakpinar@hotmail.com

Received: January 11, 2020

Revised: March 29, 2020

Accepted: April 19, 2020
Copyright $\odot 2020$ Korean Society of Interventional Neuroradiology

This is an Open Access article distributed under the terms of the Creative Commons Attribution Non-Commercial License (http://creativecommons.org/licenses/by-nc/4.0) which permits unrestricted non-commercial use, distribution, and reproduction in any medium, provided the original work is properly cited.

pISSN 2093-9043 eISSN 2233-6273 
The purpose of this study was to evaluate the efficacy and safety of direct common carotid artery puncture (DCCAP) in patients with an unaccessible femoral route for mechanical thrombectomy. We also discussed technical difficulties and pitfalls accompanied by a literature review.

\section{MATERIALS AND METHODS}

Twenty-five patients who underwent DCCAP were included, among 1,126 consecutively-collected, retrospectively-analyzed mechanical thrombectomy cases between January 2015 and April 2019 in 4 comprehensive stroke centers. Among 25 cases with carotid puncture, 4 cases were excluded due to an aborted thrombectomy attempt resulting from unsuccessful sheath placement. Demographic and angiographic data of the remaining 21 cases were extracted from the Interventional Neurology Database for analysis.

Cases were included in the study according to the following criteria: age $>18$ years, anterior circulation stroke up to 8 hours since symptom onset, prestroke modified Rankin Scale (mRS) of 0-1, large vessel occlusion demonstrated by computed tomography angiography (CTA), Alberta Stroke Program Early CT Score (ASPECTS) of $>6$, and favorable collateral status on CTA. Carotid puncture decision was taken in patients with bilateral femoral artery occlusion or with unsuccessful carotid catheterization because of anatomical barriers. For the patients with type 2-3 arch or bovine arch, we decided to switch to DCCAP after failing attempts with telescopic or exchange technique use. We assessed demographical, clinical, and time metrics information of the patients in Table 1. This study was approved by Samsun Training and Research Hospital Institutional Research Ethics Board (approval number: TUEK 1192018 GOKAEK/2-18). Written Informed Consent was obtained from next of kin.

\section{Direct common carotid artery puncture technique}

\section{Patient's position}

Before starting carotid puncture, the patient was placed in a supine position with a supporting pillow put under the nape of the neck, and with the neck taken to extension. The head was stabilized with an adhesive band on the patient's forehead area.

Local anesthesia: The area of puncture was sterilized un- der suitable conditions and $6 \mathrm{~mL} 2 \%$ prilocaine was applied subcutaneously as local anesthesia before the procedure. The patient was covered with a sterile dressing at the area of the procedure, with the patient's face left open.

Conscious sedation: Depending on operator preferences, either $25 \mathrm{mcg}$ intravenous (iv) fentanyl or/and 1 to $2 \mathrm{mg}$ iv midazolam or $25 \mathrm{mcg}$ iv fentanyl or/and 1 to $2 \mathrm{~mL}$ propofol or 0.6 mcg dexmedetomidine were administered.

\section{Puncture site}

The point that the CCA was palpated from the lateral of the cricoid cartilage, medial of sternocleidomastoid muscle, and 3 to $4 \mathrm{~cm}$ above clavicle, was determined as the puncture site. Artery palpation was easier with turning the head away from the side of puncture, with the neck in extension. In cases where the roadmap was performed via catheterization of the proximal CCA, puncture was made under a view of the roadmap; in other cases, when the roadmap could not be performed due to femoral occlusion, inability to catheterize the proximal CCA because of arch complexity or osteal lesion or aorto-iliac severe tortuosity, carotid puncture was made with manual palpation of the arterial pulse.

Like femoral puncture, we used ultrasonography only in case of difficulty in manual carotid palpation. We only used ultrasonography in 4 cases, which resulted in failed DCCAP due to an unfavorable carotid anatomy.

\section{Puncture needle and sheath}

Puncture was performed with an angle of 45 degrees by using either a 21-G micropuncture kit or standard 18-G arterial access needle, or 5 French sheath (introducer sheath 7 $\mathrm{cm}$-wire length $45 \mathrm{~cm}$ guide wire 0.018 inch) or 6/7 French sheath (introducer sheath $11 \mathrm{~cm}$-wire length $45 \mathrm{~cm}$-guide wire 0.035 inch) depending on the operators. If a 5 French sheath was used initially, it was exchanged for a 6/7 French sheath.

\section{Sheath placement to common carotid artery}

In cases where the roadmap was possible by placing a diagnostic catheter to the CCA, we could safely advance a 0.038 inch standard guidewire inside the artery. If the wire could be placed in the internal carotid artery (ICA), then we could advance the sheath to the desired position. Cases in which we could not get the roadmap via proximal CCA injection, one might try to get a roadmap from a puncture needle; however, it is not always easy to hold a puncture 


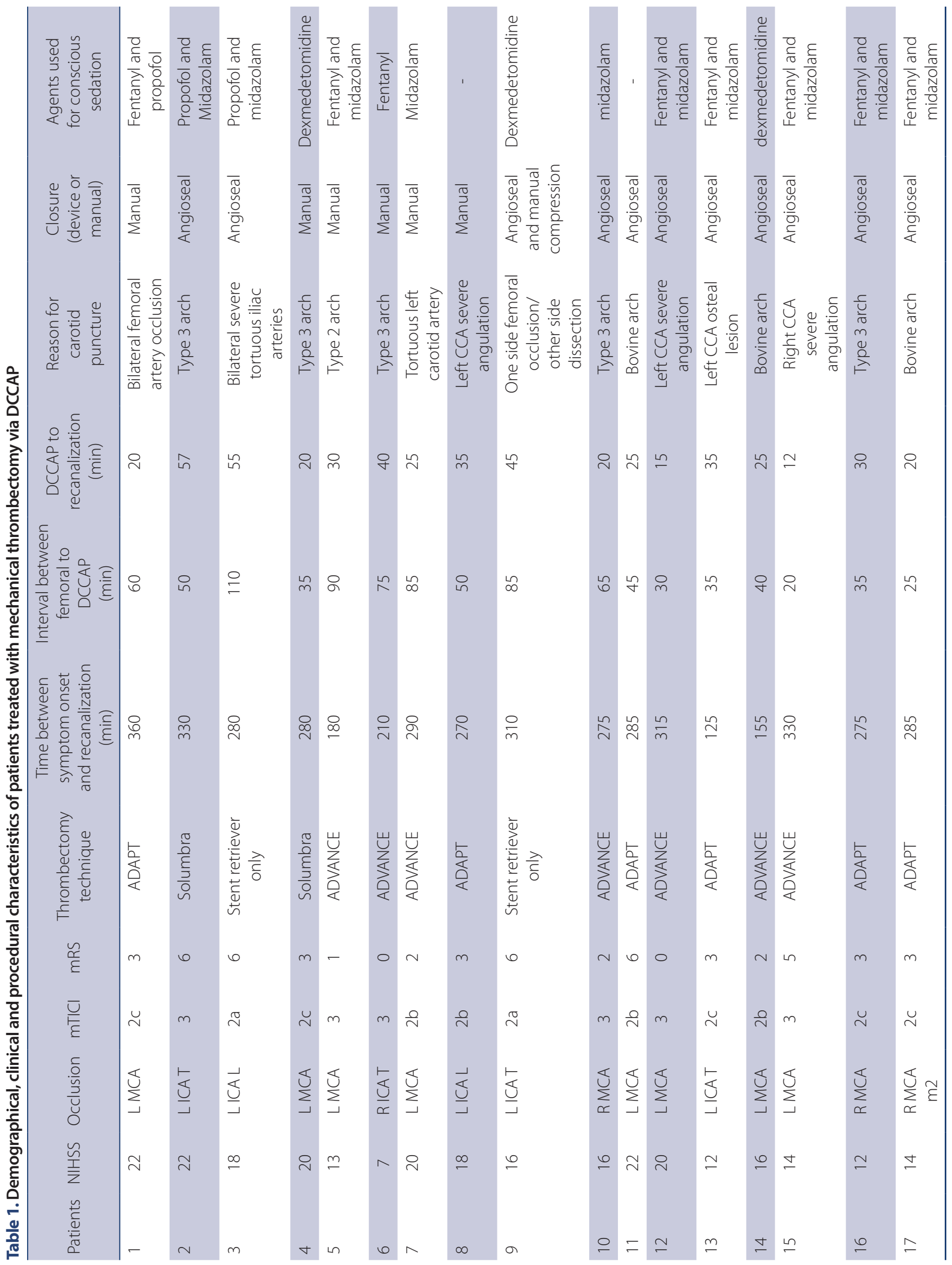




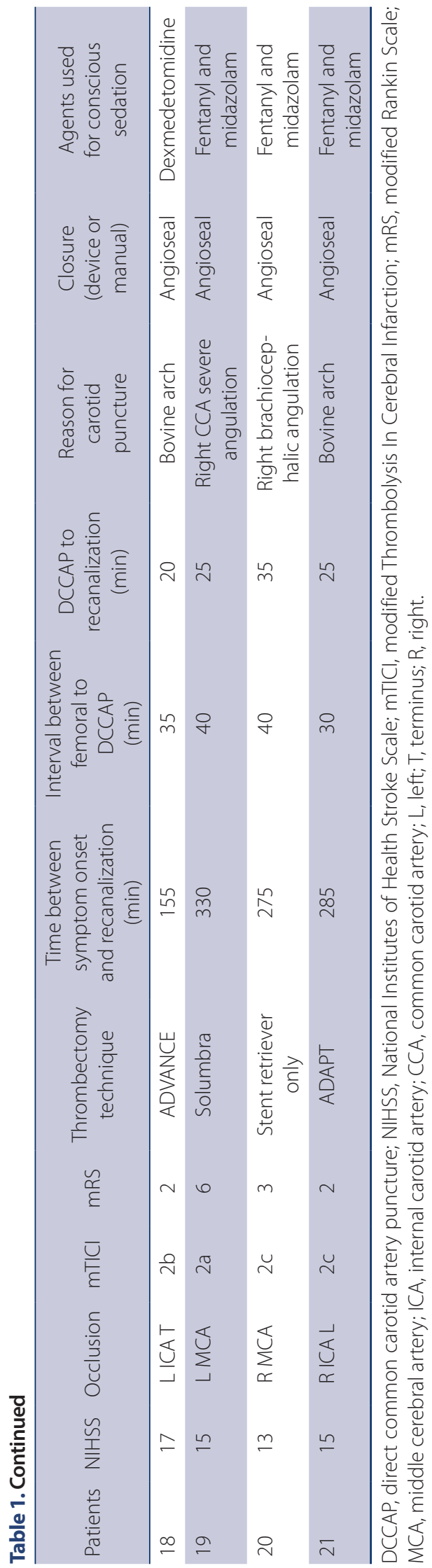

needle stable and the same time give simultaneous contrast. If the needle was stabilized and a roadmap could be taken, the method above was applied. If the roadmap through the puncture needle could not be performed, a 0.018 or 0.038 guidewire according to operator preference was inserted and advanced through the puncture needle under fluoroscopy guidance. Later, the needle was pulled back, and a dilator was moved over the guidewire. After removing the guidewire, a roadmap was taken via contast injection through the dilator. With the guidance of a j pointed 0.035 inch hydrophilic guidewire, the sheath was inserted in ICA as explained above. In total, 2,000 unit heparin was applied for all cases after sheath placement. Carotid sheath placement time was included in DCCAP to recanalization time.

\section{Mechanical thrombectomy technique}

Depending on the operator, direct aspiration, stent retriever only, ${ }^{2}$ or aspiration plus stentretriever techniques (ADAPT, ${ }^{6}$ ADVANCE, ${ }^{7}$ Solumbra ${ }^{8}$ ) were used. The ADAPT ${ }^{6}$ technique is a direct aspiration technique, whereas the ADVANCE ${ }^{7}$ and Solumbra ${ }^{8}$ techniques are a combination of aspiration and stent retriever use. The main difference between the ADVANCE and Solumbra techniques is the position of the distal access catheter. In the ADVANCE technique, the distal access catheter is advanced over the stent retriever; in Solumbra, the distal access catheter is placed proximal to the stent retriever.

\section{Sheath removal}

Closure was made with 8-F Angio-Seal ${ }^{\top \mathrm{M} M}$ (St. Jude Medical, St. Paul, MN, USA). In case of unavailability of angioseal or failure of angioseal placement, moderate pressure manual compression was applied for 15 to 20 minutes in order not to cause blockage of carotid flow.

Heparin reversal with protamine sulfate was used in all cases.

\section{RESULTS}

Within a period of 4 years, DCCAP was applied in 25 (2.2\%) cases among 1,126 patients treated with mechanical thrombectomy. Demographical, clinical, and procedural information of the patients are shown in Table 1.

Twenty-one (11 male and 10 female) patients were included with a mean age of 69 \pm 12 (median 78, 64-88) years. Median 
National Institutes of Health Stroke Scale (NIHSS) score was 16 (7-22), and median ASPECTS was 7 (5-10). IV thrombolytic therapy was given to $10(48 \%)$ of the cases. The rate of successful recanalization (modified Thrombolysis In Cerebral Infarction [mTICl] 2b-3) was 18 (86\%), and the rate of the firstpass recanalization was 9 (43\%). The rate of symptomatic hemorrhage was 2 (10\%), while the rate of asymptomatic hemorrhage was 5 (24\%). Good clinical outcome (mRS 0-2 at 90 days) was achieved in 8 (38.1\%) patients, and mortality was seen in 5 (24\%) patients. The period of time between DCCAP puncture and recanalization varied between 12 to 57 minutes. The decision time for switching to DCCAP was shortened by time (60 to 110 minutes in the first 3 years versus 20 to 40 minutes in last year).

The reasons among 21 patients with successful carotid puncture were unfavourable aortic arch in 11 (52\%) patients, CCA tortuosity in 6 (29\%) patients, bilateral iliac or femoral artery stenosis or occlusion in 3 (14\%) patients, and CCA osteal severe stenosis in 1 (5\%) patient. In 4 carotid puncture cases with unsuccessful sheath placement, 1 had severe cervical ICA stenosis, 2 had an inability to navigate from the external carotid artery to ICA due to the lower carotid bifurcation and severe carotid tortuosity, and in the last case, the procedure was halted due to neck hematoma related to recurrent puncture attempts.

Among 21 patients with successful sheath placement, closure device was used in 15 cases, and manual compression was applied in 6 cases, including 1 case with manual compression due to an unsuccessful closure device attempt. Three cases with manual compression and 1 case with closure device had experienced asymptomatic neck hematoma.

In one case, asymptomatic dissection developed in cervical ICA due to the advancement of a guidewire through a kinking ICA.

\section{DISCUSSION}

In the present study, we demonstrated that DCCAP could be a rescue treatment option for untreatable cases via a transfemoral route. In this study, we revealed that among 25 attempted DCCAP cases with unfavorable femoral or aortic arch access, 21 patients were treated via DCCAP. The rate of successful recanalization (mTICl2b-3) was 18 (86\%), and the rate of the first-pass recanalization was 9 (43\%). The rate of symptomatic hemorrhage was $2(10 \%)$, while the rate of asymptomatic hemorrhage was 5 (24\%). Good clinical outcome (mRS 0-2 at 90 days) was achieved in 8 (38\%) patients, and mortality was seen in 5 (24\%) patients.

With direct CCA puncture, intracranial aneurysm, arteriovenous malformation, carotid stenting, intracranial stenting, and mechanical thrombectomy therapies have been reported in case reports and case series. ${ }^{9-13}$ Endovascular therapy via transfemoral route failed in $1 \%$ to $6 \%$ of cases due to anatomical challenges. These include femoral occlusion, shaggy or aneurysmal aortas, previous aortic vascular surgery, type II or type III arch/elongated arch, bovine arch, osteal stenosis, tortuous carotid arteries, diffuse atherosclerosis with calcified and poor compliant vessels. ${ }^{9}$ DCCAP is an emerging treatment option for mechanical thrombectomy that is ineligible for a transfemoral approach to treat acute stroke patients. A recently conducted study showed a strong correlation between supra-aortic vessel anatomy and guiding catheter placement to the ICA. ${ }^{14}$ This means that the aortic arch type and supra-aortic vessel variations can lengthen the processing time and have a negative influence on clinical outcome. In such cases, the risk for embolic stroke or dissection also increases. ${ }^{3}$ Shorter mechanical thrombectomy procedure time has been reported to be associated with good clinical outcome. ${ }^{1}$ For these reasons, in cases with unfavorable anatomy, other alternative routes such as trans-radial, trans-brachial ${ }^{5,15}$ or direct CCA puncture can be preferred. ${ }^{16}$ The radial or brachial approach can also lengthen the duration of the process, depending on the anatomical structure and the DCCAP option should be kept in mind as an alternative. It was observed that gaining experience in carotid puncture resulted in an earlier decision for switching to DCCAP, which prevented delay for unnecessary maneuvers via the transfemoral route. For this reason, especially in cases with advanced age, the presence of signs of aortic and supra-aortic vessel abnormalities as explained above on CTA should be examined with different sections or arcus aortography should be performed, and the course of supra-aortic vessels should be assessed. In these cases, in which we assume that the transfemoral approach would take a long time, the DCCAP decision should be taken quickly, and the time of recanalization should not be delayed.

In the literature, case reports or case series with few patients treated by mechanical thrombectomy via DCCAP were reported. Successful recanalization was mostly achieved, but these articles lack detailed procedural data. ${ }^{12,17-20}$ Recently, the largest $(n=11)$ case series on this issue was reported. ${ }^{16}$ In 
this study, 10 in 11 cases had a successful carotid puncture.

Successful recanalization was obtained in $80 \%$ of 10 cases who received DCCAP, while good clinical outcome was obtained in $20 \%$. Mortality was seen in $30 \%$ of the cases. In this study, DCCAP was decided between 30 and 60 minutes and following the puncture, and successful recanalization was obtained between 14 and 42 minutes. ${ }^{16}$

In our study, successful recanalization, good clinical outcome, and mortality rates were found as $86 \%, 38 \%, 29 \%$, respectively. The period of time between the DCCAP puncture and recanalization varied between 12 to 37 minutes. The decision time for switching to DCCAP (femoral puncture to DCCAP) was 20 to 110 minutes.

Besides its advantages, DCCAP can have serious complications. The most feared complication is neck hematoma development, which can be critical if hematoma expands and causes tracheal obstruction. High dose heparin, large-scaled sheath placement, and inappropriate manual compression may increase this risk. In order to prevent neck hematoma, heparin should be neutralized, a sheath with a diameter as low as possible should be used, and manual compression should be applied in moderate intensity for a sufficient period. Although asymptomatic subcutaneous hematoma was observed, no severe neck hematoma that could be life-threatening was observed. Other complications reported in the literature were dissection at a punctured artery, sheath kinking, and pseudo-occlusion in the vessel due to severe manual compression following sheath removal resulting in a new ischemic stroke. ${ }^{16,20}$

Hemostasis at the femoral access site is generally successfully achieved using closure devices such as Angio-Seal ${ }^{\mathrm{T} M}$, Exoseal (Cordis Corporation, Bridgewater, NJ, USA) and Star Close (Abbott, Chicago, IL, USA). The use of closure devices for this technique is limited, and there is no approved closure device for cervical carotid closure. Recently, the use of closure devices after DCCAP has been reported. Feared complications of closure devices are distal embolization of collagen plug, dissection, and hematoma formation due to insufficient/unsuccessful closure. ${ }^{21}$ However, the rate of complication reported with closure devices in both our study and literature was quite low. Generally, hemorrhage in the form of leakage and subcutaneous hematoma is seen due to insufficient closure. In patients who are given more than 2000 units, protamine sulfate can be given in a suitable dose, and this complication can be prevented with mild manual compression that lasts for 2 to 3 minutes. No life-threatening complication of the closure device has been reported. We used only Angioseal because of our limited experience with other closure devices. Successful and effective hemostasis was provided in most of our cases (13 out of 15 cases) with Angioseal. In one of our cases with mild subcutaneous hematoma, the hematoma decreased in a short period after manual compression. In another case, we had to perform manual compression since the closure device could not be extended over the guidewire. No severe complication or collagen plug embolism was seen.

Although carotid puncture can be performed in each case, the sheath may not be inserted successfully. The reasons may be carotid bifurcation below the C4 level, perpendicular insertion of puncture needle to CCA leading to failure of wire distal advancement, presence of proximal ICA severe stenosis or occlusion, and presence of subcutaneous hematoma due to recurrent needle insertions. In these conditions, the assistance of ultrasonography during puncture needs to be clarified in further studies.

Limitations of this study were a relatively low number of cases and a lack of a control group for other access routes.

\section{CONCLUSION}

The DCCAP approach might be a safe and feasible rescue thrombectomy strategy for unaccessible patients via the transfemoral route. One of the most critical points is a timely decision for switching to DCCAP.

\section{Fund}

None.

\section{Ethics Statement}

This study was approved by the institutional research ethics board.

\section{Conflicts of Interest}

The authors have no conflicts to disclose.

\section{Author Contribution}

Concept and design: CKA and EG. Analysis and interpretation: CKA, EG, AOO, and SG. Data collection: CKA, EG, OA, YI, $\mathrm{SG}$, and $\mathrm{AOO}$. Writing the article: $C K A, E G$, and $\mathrm{AOO}$. Critical revision of the article: $C K A, E G$, and $A O O$. Final approval of the article: CKA and EG. Statistical analysis: CKA. Obtained 
funding: none. Overall responsibility: CKA, EG, OA, YI, SG, and AOO.

\section{ORCID}

Cetin Kursad Akpinar: https://orcid.org/0000-0001-9512-1048

Erdem Gurkas: https://orcid.org/0000-0001-8086-2900

Ozlem Aykac: https://orcid.org/0000-0003-4987-0050

Yusuf Inanc: https://orcid.org/0000-0003-2652-1157

Semih Giray: https://orcid.org/0000-0002-0722-3181

Atilla Ozcan Ozdemir: https://orcid.org/0000-0002-9864-6904

\section{REFERENCES}

1. Spiotta AM, Vargas J, Turner R, Chaudry MI, Battenhouse H, Turk AS. The golden hour of stroke intervention: effect of thrombectomy procedural time in acute ischemic stroke on outcome. J Neurointerv Surg 2014;6:511-516

2. Goyal M, Demchuk AM, Menon BK, Eesa M, Rempel JL, Thornton J, et al.; ESCAPE Trial Investigators. Randomized assessment of rapid endovascular treatment of ischemic stroke. N Engl J Med 2015;372:1019-1030

3. Ribo M, Flores A, Rubiera M, Pagola J, Mendonca N, Rodriguez-Luna $D$, et al. Difficult catheter access to the occluded vessel during endovascular treatment of acute ischemic stroke is associated with worse clinical outcome. J Neurointerv Surg 2013;5 Suppl 1:i70-i73

4. Oselkin M, Satti SR, Sundararajan SH, Kung D, Hurst RW, Pukenas BA. Endovascular treatment for acute basilar thrombosis via a transradial approach: initial experience and future considerations. Interv Neuroradiol 2018;24:64-69

5. Okawa M, Tateshima S, Liebeskind D, Ali LK, Thompson ML, Saver J, et al. Successful recanalization for acute ischemic stroke via the transbrachial approach. J Neurointerv Surg 2016;8:122-125

6. Turk AS, Frei D, Fiorella D, Mocco J, Baxter B, Siddiqui A, et al. ADAPT FAST study: a direct aspiration first pass technique for acute stroke thrombectomy. J Neurointerv Surg 2014;6:260-264

7. Gurkas E, Akpinar CK, Aytac E. ADVANCE: an effective and feasible technique in acute stroke treatment. Interv Neuroradiol 2017;23:166-172

8. Delgado Almandoz JE, Kayan Y, Young ML, Fease JL, Scholz JM, Milner AM, et al. Comparison of clinical outcomes in patients with acute ischemic strokes treated with mechanical thrombectomy using either Solumbra or ADAPT techniques. J Neurointerv Surg 2016;8:1123-1128

9. Dorfer C, Standhardt H, Gruber A, Ferraz-Leite H, Knosp E,
Bavinzski G. Direct percutaneous puncture approach versus surgical cutdown technique for intracranial neuroendovascular procedures: technical aspects. World Neurosurg 2012;77:192200

10. Blanc R, Piotin M, Mounayer C, Spelle L, Moret J. Direct cervical arterial access for intracranial endovascular treatment. Neuroradiology 2006;48:925-929

11. Samaniego EA, Dabus G, Raju R, Tsoukas Al, Linfante I. Intracranial angioplasty and stenting through direct carotid puncture. J Neuroimaging 2013;23:207-210

12. Mokin M, Snyder KV, Levy El, Hopkins LN, Siddiqui AH. Direct carotid artery puncture access for endovascular treatment of acute ischemic stroke: technical aspects, advantages, and limitations. J Neurointerv Surg 2015;7:108-113

13. Jadhav AP, Ribo M, Grandhi R, Linares $G$, Aghaebrahim A, Jovin TG, et al. Transcervical access in acute ischemic stroke. J Neurointerv Surg 2014;6:652-657

14. Kaymaz ZO, Nikoubashman O, Brockmann MA, Wiesmann M, Brockmann C. Influence of carotid tortuosity on internal carotid artery access time in the treatment of acute ischemic stroke. Interv Neuroradiol 2017;23:583-588

15. Sur S, Snelling B, Khandelwal P, Caplan JM, Peterson EC, Starke RM, et al. Transradial approach for mechanical thrombectomy in anterior circulation large-vessel occlusion. Neurosurg Focus 2017:42:E13

16. Roche A, Griffin E, Looby S, Brennan P, O'Hare A, Thornton J, et al. Direct carotid puncture for endovascular thrombectomy in acute ischemic stroke. J Neurointerv Surg 2019;11:647-652

17. Cilingiroglu M, Hakeem A, Wholey M, Goktekin O. Direct carotid access for endovascular management of acute ischaemic stroke. Eurolntervention 2017;13:e1120-e1121

18. Benichi S, Consoli A, Coskun O, Boulin A, Wang A, Rodesch G, et al. Thrombectomy by direct cervical access in a case of common carotid occlusion with collateral supply to the internal carotid artery: an uncommon anatomical variant. World Neurosurg 2019;124:84-86

19. Roche AD, Murphy B, Adams N, Sheahan R, Brennan P, Looby S. Direct common carotid artery puncture for endovascular treatment of acute large vessel ischemic stroke in a patient with aortic coarctation. J Stroke Cerebrovasc Dis 2017;26:e211-e213

20. Kawabori M, Osanai T, Goto S, Iwasaki M, Niiya Y, Mabuchi S, et al. Direct common carotid artery puncture for acute thrombectomy against ischemic stroke. J Neurosurg Sci 2018;62:612-614

21. Cuellar H, Guimaraens L, Ambekar S, Vivas E, Theron J. Angiosea ${ }^{\mathrm{TM}}$ as a hemostatic device for direct carotid puncture during endovascular procedures. Interv Neuroradiol 2015;21:273-276 